\title{
Optimization of Conditions for Organic Acid Extraction from Edible Plant Material as Applied to Radish Sprouts
}

\author{
Joanna Chlopicka • Justyna Dobrowolska-Iwanek • \\ Michal Wozniakiewicz • Pawel Zagrodzki
}

Received: 18 July 2013 / Accepted: 31 October 2013 / Published online: 27 November 2013

(C) The Author(s) 2013. This article is published with open access at Springerlink.com

\begin{abstract}
In recent years, there has been growing interest in the influence of sprouts on health. Fruit and vegetables are the main sources of organic acids for humans; however, little is yet known about organic acids in sprouts. In this study, the selection of the optimal parameters for extraction of organic acids from fresh, edible sprouts is reported. Two extraction techniques: microwave-assisted (MAE) and ultrasoundassisted were compared. The experimental conditions were optimized in terms of extraction time, temperature, and composition of extraction solution. To determine the influence of time and temperature of extraction or sample cooling, solvents used for extraction, on the analytical signal in isotachophoretic separation, the methods of experimental planning fractional factorial design: $3^{\mathrm{k}-1}$ were used (three factor, three-level design). The optimal conditions for extraction of organic acids from radish sprouts were MAE, $90{ }^{\circ} \mathrm{C}$; $18 \mathrm{~min}$; and $0.01 \mathrm{M} \mathrm{NaOH}$ as a solvent.
\end{abstract}

Keywords Microwave-assisted extraction · Ultrasound-assisted extraction $\cdot$ Extraction conditions $\cdot$ Organic acids $\cdot$ Sprouts

J. Chlopicka $(\bowtie) \cdot J$. Dobrowolska-Iwanek $\cdot$ P. Zagrodzki

Department of Food Chemistry and Nutrition, Medical College, Jagiellonian University, 9 Medyczna St., 30-688 Krakow, Poland e-mail: chlopicka@gmail.com

M. Wozniakiewicz

Laboratory for Forensic Chemistry, Department of Analytical

Chemistry, Jagiellonian University, 3 Ingardena St.,

30-060 Krakow, Poland

P. Zagrodzki

Department of Nuclear Physical Chemistry,

Henryk Niewodniczanski Institute of Nuclear Physic,

152 Radzikowskiego St., 31-342 Krakow, Poland

\section{Introduction}

Conversion of seeds into sprouts through germination results in many biochemical changes. It increases the levels of free amino acids, flavonoids, fatty acids, and water-soluble vitamins; therefore, the nutritional value of sprouts is higher than that of respective seeds. However, little is known about the quantity and types of organic acids in different types of vegetable sprouts. It is essential to determine the concentrations of organic acids in edible sprouts in order to be able to classify them as a functional food. For example, sprouted buckwheat grains may constitute a valuable component of any healthy diet (Koyama et al. 2013; Kim et al. 2004).

In recent years, we have observed progress in the development and application of new techniques for fast and efficient extraction of organic compounds from different plant materials (Alupului et al. 2009; Mandal et al. 2007; Gallo et al. 2010). Microwave-assisted extraction (MAE) is one of the most important techniques used for isolation of various compounds from plants or vegetal materials for both analytical and industrial purposes (Zhang et al. 2011; Liu et al. 2011; Fang et al. 2012). MAE is based on the interaction between microwaves generated by a magnetron or another radiation source and the sampleextracting solvent system.

This technique offers high extraction efficiency, low time and reagent consumption, and is relatively rapid. Moreover, it might be applied for extraction of water soluble and insoluble plant components. However, the microwave extraction process requires several factors to be optimized like power of microwave irradiation, time of exposure, moisture content and pulverization of sample, type and composition of extraction solvent, sample-to-solvent ratio, and extraction temperature and pressure (Wang and Weller 2006). Moreover, the physicochemical properties of the extracted substances strongly 
affect the extraction yield, as microwave energy is absorbed only by polar particles. Thus, in the case of extraction of nonpolar compounds, the choice of extracting solvent should also be based by its ability to absorb microwave energy (Zhang et al. 2011). MAE has a broad application range. It was used for the extraction of different substances from food and plant material, for instance, phenolic acids from Salviae Miltiorrhizae Radix (Fang et al. 2012), ascorbic acid, and other antioxidants from green pepper and guava (Yu et al. 2009; Xiao et al. 2009), alkaloids, e.g., caffeine from tea leaves (Pan et al. 2003), and many others (Chan et al. 2011). To the best of the authors' knowledge, the present report is the only published paper presenting the use of the MAE technique for organic acid isolation; thus, until now, there has been none describing the application of MAE for isolation of such compounds from vegetable material.

The aim of this study was to select the optimal parameters for the extraction of organic acids from vegetable material on the example of radish sprouts. The following experimental conditions were optimized: extraction time, temperature, and composition of extraction solution. Two extraction techniques, microwave-assisted extraction and ultrasound-assisted extraction (UAE) were compared in terms of extraction efficiency. The evaluation was performed using isotachophoretic separation and determination of seven organic acids (tartaric acid, citric acid, malic acid, lactic acid, succinic acid, acetic acid, and oxalic acid), as the main acids present in vegetables and fruit.

\section{Materials and Methods}

Sample fresh radish sprouts were purchased at a local store.

\section{Reagents}

Deionized water $18 \mathrm{M} \Omega \mathrm{cm}$ was obtained using a Milli Ro \& Q water purification system (USA). The following organic acid standards were used: tartaric acid and $80 \%$ lactic acid from POCh (Gliwice/Poland); monohydrate citric acid and oxalic acid from Chempur (Piekary Śląskie/Poland); and DLmalic acid and succinic acid from Lancaster (Morecambe England) and acetic acid (99.5\%) from Chempur (Piekary Ślaskie, Poland). Methylhydroxyethylcellulose (M-HEC) was purchased from HERCULES (Prague/Czech Republic), alanine from LACHEMA (Brno/Czech Republic), caproic acid from Fluka (Steinheim/Germany), L-histidine was from Serva (Heidelberg Germany), hydrochloric acid (36-38\%) was from Baker Analyzed, and sodium hydroxide (30 \%) aqueous solution was from POCh (Gliwice/Poland). All chemicals were of analytical purity. Phosphate buffer $50 \mathrm{mM}$, pH7.4 (Ph.B.) was prepared by adding $0.16 \mathrm{~g}$ of monosodium phosphate monohydrate and $1.04 \mathrm{~g}$ of disodium phosphate (POCh, Gliwice/Poland) heptahydrate to $100 \mathrm{ml}$ of water. The recipe of buffer solution was verified by $\mathrm{pH}$ measuring.

\section{Apparatus}

Isotachophoretic separation was performed using an Electrophoretic Analyser EA202M (Villa Labeco, Slovakia) with conductivity detection. The system was equipped with a sample valve of $30 \mu \mathrm{m}$ fixed volume and two capillaries: the preseparation capillary (ID $90 \mathrm{~mm} \times 0.9 \mathrm{~mm}$ ) and analytical capillary (ID $160 \mathrm{~mm} \times 0.3 \mathrm{~mm}$ ). The current in the preseparation column was $250 \mu \mathrm{A}$, and in the analytical column, initially, $60 \mu \mathrm{A}$ which during detection was reduced to $50 \mu \mathrm{A}$. Hydrochloric acid $(10 \mathrm{mM})$ containing $0.2 \%$ M-HEC adjusted to $\mathrm{pH} 3.5$ with $\beta$-alanine was the leading electrolyte. The terminating electrolyte contained $5 \mathrm{mM}$ caproic acid and $5 \mathrm{mM}$ histidine.

A MARS X microwave-oven (CEM, Matthews, USA) equipped with 14 pressurized $55 \mathrm{~mL}$ volume MarsXpress ${ }^{\circledR}$ PFA vessels was used for MAE process. The internal system of the device allowed for temperature control up to $240^{\circ} \mathrm{C}$ and power up to 1,200 W. Ultrasonic homogenizer VC 505(Sonics \& Materials, USA) was used for ultrasound-assisted extraction from biological material.

\section{Sample Preparation}

Radish sprouts were dried at $50^{\circ} \mathrm{C}$ for $2 \mathrm{~h}$ in a ventilated oven. For each experiment, $500.0 \pm 0.5 \mathrm{mg}$ of the dried sprouts were weighted using an analytical balance. Samples were transferred into extraction vessels: MarsXpress and Falcon vials $(25 \mathrm{~mL})$ for UAE. Then, the content of each vessel was immersed in the appropriate extracting solution. The PFA vessels were then tightly twisted, as the MAE was performed in a closed system, whereas the UAE technique was performed in the open mode as the ultrasonic probe was introduced through the vial opening. Samples prepared in such a way were ready for further extraction procedure. All experiments were performed in triplicate.

After extractions, the vessels were cooled down to the room temperature. The extracts were transferred to polypropylene vials. Additionally, sprout residues were rinsed with $1 \mathrm{ml}$ of appropriate solvent and the collected rinsing solution was pooled with the sample extract. Finally, extracts were stored in darkness at $-80^{\circ} \mathrm{C}$ until further analysis.

\section{Experimental Design and Statistical Approach}

To determine the influence of a number of experimental parameters (time and temperature of extraction or sample cooling, solvents used for extraction) on the sample response 
(analytical signal in isotachophoretic separation), the methods of experimental planning were used (Prvana and Streetb 2002; Bisgaard 1996; McLean and Anderson 1984).

The optimization of the parameters (factors) affecting the final concentrations of organic acids was carried out following the factorial design of experiments. The factors applied by the experimenter are called treatment factors and the choice of their levels (the possible values that they can take) is made by considering specific conditions for each parameter, but still remains arbitrary to some degree. The sample response measured in our study was analytical signal in isotachophoretic separation, and the treatment factors chosen as having any influence on it were time, temperature (of extraction or sample cooling), and type of solvents used for extraction. The full three-level factorial design (three factors, each run at three levels) was considered uneconomical, as it required 27 $\left(3^{3}\right)$ trials, i.e., many more than to identify most relevant parameters and their levels. Therefore, as an alternative, the screening of experimental parameters was carried out using fractional factorial design of experiments: $3^{1}$ in which we selected $1 / 3$ fraction of full $3^{3}$ design with only nine trials (nine different combinations of treatment factors) still efficient for our purposes. The experimental matrix and scheme of factor coding in microwave extraction, comprising parameter values and coded levels $(-1,0,+1)$ is shown in Table 1 .

The time and temperature of an extraction process as well as the kind of extracting solution were chosen as major factors affecting extraction yield. It was previously proven that the temperature and time of exposure to microwaves played a significant role in microwave-assisted extraction. The MAE technique requires working at an elevated temperature; thus, 50,70 , and $90^{\circ} \mathrm{C}$ were chosen as the tested temperature levels. It was found that work at a stable temperature lower than $50{ }^{\circ} \mathrm{C}$ was not possible while working at a temperature higher than $90{ }^{\circ} \mathrm{C}$ might cause some loss of analytes due to decay of compounds. Microwave-assisted technique may be highly efficient even when performed for a very short time (Alupului et al. 2009; Mandal et al. 2007); thus, 2 min was tested as the minimal value of the second parameter, i.e., extraction time. Then, two other levels were tested with a step of $8 \mathrm{~min}$.

In the case of the ultrasound-assisted extraction, three methods of sample cooling during the extraction were tested, as the ultrasonic probe generates significant amount of heat in the sample-extraction solvent system. First, an ice bath was

Table 1 Parameter values and coded sets of experimental conditions for MAE and UAE experiments

\begin{tabular}{|c|c|c|c|c|c|c|c|}
\hline \multirow[b]{2}{*}{ No. ${ }^{\mathrm{d}}$} & \multirow[b]{2}{*}{ Extraction technique } & \multicolumn{2}{|l|}{ Time $[\mathrm{min}]$} & \multicolumn{2}{|l|}{ Temperature $\left[{ }^{\circ} \mathrm{C}\right]$} & \multicolumn{2}{|l|}{ Extracting solution } \\
\hline & & Parameter value & Code & Parameter value & Code & Parameter value & Code \\
\hline 1 & MAE & 2 & -1 & 50 & -1 & Water & -1 \\
\hline 2 & MAE & 10 & 0 & 50 & -1 & $0.01 \mathrm{M} \mathrm{NaOH}$ & 1 \\
\hline 3 & MAE & 18 & 1 & 50 & -1 & $50 \mathrm{mM}$ Ph.B. pH 7.4 & 0 \\
\hline 4 & MAE & 2 & -1 & 70 & 0 & $0.01 \mathrm{M} \mathrm{NaOH}$ & 1 \\
\hline 5 & MAE & 10 & 0 & 70 & 0 & 50 mM Ph.B. pH 7.4 & 0 \\
\hline 6 & MAE & 18 & 1 & 70 & 0 & Water & -1 \\
\hline 7 & MAE & 2 & -1 & 90 & 1 & 50 mM Ph.B. pH 7.4 & 0 \\
\hline 8 & MAE & 10 & 0 & 90 & 1 & Water & -1 \\
\hline 9 & MAE & 18 & 1 & 90 & 1 & $0.01 \mathrm{M} \mathrm{NaOH}$ & 1 \\
\hline 10 & UAE & 1 & -1 & $15^{\mathrm{a}}$ & -1 & Water & -1 \\
\hline 11 & UAE & 5 & 0 & $15^{\mathrm{a}}$ & -1 & $0.01 \mathrm{M} \mathrm{NaOH}$ & 1 \\
\hline 12 & UAE & 15 & 1 & $15^{\mathrm{a}}$ & -1 & 50 mM Ph.B. pH 7.4 & 0 \\
\hline 13 & UAE & 1 & -1 & $25^{\mathrm{b}}$ & 0 & $0.01 \mathrm{M} \mathrm{NaOH}$ & 1 \\
\hline 14 & UAE & 5 & 0 & $25^{\mathrm{b}}$ & 0 & 50 mM Ph.B. pH 7.4 & 0 \\
\hline 15 & UAE & 15 & 1 & $25^{\mathrm{b}}$ & 0 & Water & -1 \\
\hline 16 & UAE & 1 & -1 & $40^{\mathrm{c}}$ & 1 & 50 mM Ph.B. pH 7.4 & 0 \\
\hline 17 & UAE & 5 & 0 & $40^{\mathrm{c}}$ & 1 & Water & -1 \\
\hline 18 & UAE & 15 & 1 & $40^{\mathrm{c}}$ & 1 & $0.01 \mathrm{M} \mathrm{NaOH}$ & 1 \\
\hline
\end{tabular}

${ }^{\text {a }}$ Average temperatures for ice bath cooling

${ }^{\mathrm{b}}$ Average temperatures for water bath cooling

${ }^{\mathrm{c}}$ Average temperatures for air cooling

${ }^{\mathrm{d}}$ No. of factorial set 
used to keep the extraction temperature around $15^{\circ} \mathrm{C}$. A water bath was of course less effective resulting in the average temperature of $25^{\circ} \mathrm{C}$ while air cooling resulted in a substantial elevation of temperature up to $40^{\circ} \mathrm{C}$. The temperature was not stable and increased during ultrasound-assisted extraction but was carefully controlled. The effect of the time of exposure to ultrasounds on extraction yield was tested at three levels starting from $1 \mathrm{~min}$. In longer exposure time, some changes in analyte concentration were observed during preliminary experiments, and they could be caused by both analyte and/ or water evaporation. The longest time of $15 \mathrm{~min}$ might result in lowering of the amount of volatile analytes (such as acetic acid) when the extraction was performed in an open system.

Three extracting solutions were proposed for both microwave-assisted extraction and ultrasound-assisted extraction. The sodium hydroxide solution was chosen as sodium salts of acids of interest are water soluble and tend to dissociate which is important for isotachophoretic determination. Water, the most commonly used solvent, was utilized as the second extracting solution. Finally, $50 \mathrm{mM}$ phosphate buffer $\mathrm{pH} 7.4$ was applied to perform the extraction at almost neutral $\mathrm{pH}$ but at elevated ionic strength.

The raw values of analytical signals obtained for different acids under different conditions of extraction were standardized by dividing them by respective mean values for each organic acid calculated over all factorial sets for a particular type of extraction, i.e., either MAE or UAE. Then, the standardized results were scored according to the following scale: the best result for a given acid was coded by 3 , intermediate result -2 and the worst result -1 . The results for all acids and all tested conditions were summarized, and the best factorial set for each type of extraction was selected.

\section{Results and Discussion}

The purpose of our study was to select optimal parameters of extraction procedure for seven organic acids, and to this aim, the MAE and UAE methods were developed using radish sprouts as a model plant material. To make the method general, the experimental planning and factorial design was applied. The results were shown in Table 2. Generally, the highest extraction yields were obtained using water or $\mathrm{NaOH}$ solution. The best conditions corresponding to the extraction such as an answer to the question is the main factor affecting the best extraction. Since sodium hydroxide is highly an aggressive chemical, alkaline hydrolysis of organic acids residues in large molecular structures can also contribute to an increase in the concentration of small molecular acids in the extract. Thus, such process applied should be named as a hydrolytic or deep extraction.

The use of phosphate buffer resulted in low extraction efficiency. This can be exemplified via set no. 7 (or 14), where signals for three analytes were not detected. For both extraction techniques, the extraction yield for all organic acids seems to increase with temperature of process except of acetic acid.

The comparison of results (Table 2) revealed the highest score for the factorial set no. 9 in MAE. In the UAE method, set no. 17 gave the highest score, but this set failed to extract
Table 2 Standardized scores for all sets of parameters of extractions

\footnotetext{
${ }^{a}$ No. of factorial set

${ }^{\mathrm{b}}$ The best parameter set for all tested organic acids extraction
}

\begin{tabular}{|c|c|c|c|c|c|c|c|c|}
\hline \multirow[t]{2}{*}{ No. ${ }^{a}$} & \multicolumn{7}{|c|}{ Organic acid } & \multirow[t]{2}{*}{ Sum of score } \\
\hline & Tartaric & Citric & Malic & Lactic & Succinic & Acetic & Oxalic & \\
\hline 1 & 0.15 & 0.00 & 0.01 & 0.45 & 0.09 & 0.88 & 0.10 & 1.69 \\
\hline 2 & 0.00 & 0.11 & 0.02 & 0.00 & 0.43 & 1.03 & 0.24 & 1.83 \\
\hline 3 & 3.02 & 2.27 & 0.96 & 0.00 & 4.31 & 0.28 & 1.29 & 12.14 \\
\hline 4 & 2.74 & 1.36 & 2.56 & 0.69 & 2.47 & 1.00 & 2.25 & 13.07 \\
\hline 5 & 0.00 & 2.59 & 2.32 & 0.00 & 0.00 & 0.00 & 1.14 & 6.05 \\
\hline 6 & 2.39 & 0.69 & 1.26 & 1.28 & 0.96 & 1.77 & 0.19 & 8.53 \\
\hline 7 & 0.00 & 4.11 & 2.83 & 0.00 & 3.27 & 0.00 & 2.20 & 12.42 \\
\hline 8 & 0.91 & 1.06 & 1.60 & 1.05 & 1.40 & 1.14 & 0.24 & 7.40 \\
\hline $9^{\mathrm{b}}$ & 2.16 & 3.52 & 2.84 & 1.82 & 2.16 & 1.25 & 1.51 & 15.25 \\
\hline 10 & 0.54 & 0.00 & 0.06 & 0.74 & 0.00 & 0.25 & 0.00 & 1.59 \\
\hline 11 & 0.07 & 0.14 & 0.05 & 0.20 & 0.13 & 0.19 & 0.17 & 0.94 \\
\hline 12 & 0.59 & 0.00 & 0.09 & 1.67 & 0.00 & 1.01 & 2.27 & 5.62 \\
\hline 13 & 0.00 & 0.12 & 0.04 & 0.03 & 0.07 & 0.18 & 0.15 & 0.59 \\
\hline 14 & 1.09 & 0.00 & 0.21 & 0.00 & 0.00 & 0.57 & 3.89 & 5.75 \\
\hline 15 & 0.17 & 0.12 & 0.44 & 1.01 & 0.54 & 0.27 & 0.12 & 2.68 \\
\hline 16 & 0.00 & 0.00 & 0.16 & 0.00 & 0.00 & 4.57 & 0.00 & 4.73 \\
\hline 17 & 1.12 & 0.52 & 0.24 & 8.26 & 0.34 & 2.91 & 0.00 & 13.38 \\
\hline 18 & 3.05 & 1.40 & 2.31 & 0.81 & 1.81 & 0.70 & 2.25 & 12.34 \\
\hline
\end{tabular}


oxalic acid. Thus, the set 18 of slightly lower score can be a better choice for extraction all tested acids using this technique. The above example shows that sum of scores should not be evaluated only numerically, but for all compounds or selected one of particular interest.

In conclusion, the analysis of seven organic acids extracted from sprouts proved that MAE technique was more efficient compared to UAE. The optimal conditions for MAE were $90{ }^{\circ} \mathrm{C}, 18 \mathrm{~min}$, and $0.01 \mathrm{M} \mathrm{NaOH}$ as a solvent. The proposed method is of practical application and could be suitable for extraction of organic acids from sprouts of different kinds of plants and other vegetable material. This method is general and could be easily adapted to the other materials. However, the morphology and physiology of the plant tissue should be similar; otherwise, the procedure should be optimized.

Conflict of Interest Joanna Chlopicka declares that she has no conflict of interest. Justyna Dobrowolska-Iwanek declares that she has no conflict of interest. Michał Woźniakiewicz declares that he has no conflict of interest. Paweł Zagrodzki declares that he has no conflict of interest. This article does not contain any studies with human or animal subjects.

Open Access This article is distributed under the terms of the Creative Commons Attribution License which permits any use, distribution, and reproduction in any medium, provided the original author(s) and the source are credited.

\section{References}

Alupului A, Calinescu I, Lavric V (2009) AIDIC Conference Series 9:18. doi:10.3303/ACOS0909001

Bisgaard S (1996) Center for quality and productivity improvement. University of Wisconsin, Wisconsin, Report no. 148

Chan C, Yusoff R, Ngoh G, Kung FWL (2011) J Chromatogr A 1218(37):6213-25

Fang X, Wang J, Zhang S, Zhao Q, Zheng Z, Song Z (2012) Sep Purif Techn 86:149-156

Gallo M, Ferracane R, Graziani G, Ritieni A, Fogliano V (2010) Molecules 15(9):6365-6374

Kim SL, Kim SK, Park CH (2004) Food Res Inter 37(4):319327

Koyama M, Nakamura C, Nakamura K (2013) J Food Sci Techn 50(1): 86-93. doi:10.1007/s13197-011-0316-1

Liu T, Sui X, Zhang R, Yang L, Zu Y, Zhang L, Zhang Y et al (2011) J Chromatogr A 1218(47):8480-9

Mandal V, Mohan Y, Hemalatha S (2007) Pharmacogn Rev 1:7-18

McLean AI, Anderson VL (1984) Applied factorial and fractional designs. Marcel Dekker, New York

Pan X, Niu G, Liu H (2003) Chem Eng Process 42:129-133

Prvana T, Streetb DJ (2002) J Statist Plann Inference 106:245-269

Wang L, Weller CL (2006) Trends Food Sci Techn 17(6):300-12

Xiao X, Wang J, Wang G, Wang J, Li G (2009) J Chromatogr A 1216(51):8867-73

Yu Y, Chen B, Chen Y, Xie M, Duan H, Li Y, Duan G (2009) J Sep Sci 32(23-24):4227-33

Zhang H, Yang X, Wang Y (2011) Trends Food Sci Techn 22(12):672-88 